\title{
Effect of planting methods and irrigation intervals on productivity of some bread wheat cultivars
}

\author{
Al-Zahy A. A. , Al-Aref K. A. O., Khalifa Y. A. M., Ahmed H. A. \\ Department of Agronomy, Faculty of Agriculture, Al-Azhar University (Assiut Branch), Assiut, Egypt
}

\begin{abstract}
A field experiment was carried out at Experimental Farm, in Al. kalahyn, Al. Hajer village, Qeft, Qena governorate, Egypt during 2017/2018 and 2018/2019 seasons, to evaluate of three planting methods (Drilling, broadcasting and furrows) and three irrigation intervals $(21,28$ and 35 days) on productivity of three wheat cultivars ( Sids 12 -Misr 2 and Giza 168 ). The results showed that exposing wheat plants to drought stress by increasing irrigation intervals significantly decrease (1000-grain weight, grains spike weight, number of spikes $/ \mathrm{m}^{2}$, grain yield $(\mathrm{ardab} / \mathrm{fed})\left(\operatorname{argab}=150 \mathrm{Kg}\right.$, fed $=$ feddan $=4200^{2} \mathrm{~m}^{2}=0.420$ hectares $=1.037$ acres $)$, straw yield (ton/fed) and protein \%). Irrigation intervals showed highly significant difference in for all characters studies. The highest value resulted from application irrigation intervals at 21 day compare with the other irrigation intervals. Planting methods significantly affected by all a previous characters. The highest value for all studies characters resulted from using drilling method. There were highly significant differences among wheat cultivars for all characters under study. The wheat cultivar sids-12 gave of the highest values of all traits compared to the other wheat cultivars used in both seasons.
\end{abstract}

Keywords: wheat, irrigation intervals, planting methods, cultivar, yield. 


\section{Introduction}

Wheat (Triticum aestivum L.) is one of the leading cereals in the world. It belongs to the family Graminede, wheat is considered one of the most vital cereal crops not only in Egypt, but also in the whole world. As it is used for nourishment and food it supplies $20 \%$ calories and $21 \%$ of protein of the food for world people (Braune et al., 2010). In Egypt, wheat is the main winter cereal crop and is widely distributed all over the country. The cultivated area is 3. 36 million feddan with an average grain yield of $18.42 \mathrm{ardab} / \mathrm{fed}(\mathrm{argab}=150 \mathrm{Kg}$, fed $=$ feddan $=42002 \mathrm{~m}^{2}=0.420$ hectares $=1.037$ acres), with the actual local production is about 9.63 million tons and consumption is 16 million tons (FAO, 2016). From the aforementioned data the local production of wheat is not sufficient to supply the maul demand of the increasing population. This caused gap between production and consumption approximate 6 million ton that imported annually to meet the needs of local markets. So, increasing wheat production is most important possibility for reducing the gap and reaching to self sufficiency of wheat production. In this regard, researchers make efforts to increase the productivity per unit area of wheat by devising new cultivars of high productivity and low water consumption (El-Gabry and Hashem, 2018). Irrigation could be considered the limiting factor affecting crop production and agricultural expansion. In Egypt, especially in Nile Valley and Delta region, where farmers use extra water to irrigate their farms. So, irrigation optimization, i.e. applying the irrigation water timely and quantitatively will increase wheat yield and save considerable amount of water. Many researchers proved the importance of irrigation treatment to maximize wheat productivity. Sharsher and El-sayed (2000) showed that plant height, number of spike $/ \mathrm{m}^{2}$, grain yield, straw yield, number of grain /spike and 1000-grain weight were significantly affected by water stress. Also, Moagedi et al. (2010) reported that plant height, number of grain/spike, 1000-grain weight, harvest index and grain yield were significantly affected by water defecate. Moreover, planting method is one of the factors affecting on the yield of wheat. So, standard planting method of wheat has a beneficial role in affecting wheat plants distribution in the field as well as water and nutrients use efficiencies for grown plants and subsequent optimum yields. Planting method in wheat like other crops responds greatly to various agro management practices, and it affected to yield and its characters (Attia et al., 2013). The objectives of this study were: (1) Saving the amount of water by determine the number of times the irrigation needed to produce the best crop from grain yield. (2) Selection the best planting methods that gave the highest grain yield and saving the amount of seed.

\section{Materials and methods}

Two field experiments were carried out at Experimental Farm, in Al-kalahyn, $\mathrm{Al}$. Hajer village, Qeft center, Qena governorate, Egypt during seasons of $2017 / 2018$ and $2018 / 2019$, to study the 
evaluate of planting methods and number of irrigation times on productivity of three bread wheat cultivars. Studied factors: Three wheat varieties (Sids 12, Misr 2 and Giza 168) and three irrigation intervals (21day, 28 day and 35 day) and three planting methods (Drilling, furrows and broadcast). The performed experiment was designed as randomized complete block design with split- split plot arrangement of treatments with three replications. Irrigation intervals were assigned to the main plot, plant method was distributed randomly in the sub plots and wheat varieties were located in the sub- sub plots. The experimental unit area was $3.5 \mathrm{~m}$ length $\times 3.0 \mathrm{~m}$ width $\left(10.5 \mathrm{~m}^{2}\right)$. In this study, sowing date was on $17^{\text {th }}$ and $20^{\text {th }}$ November in the first and second seasons, respectively (Table 1-3). As harvested in $5^{\text {th }}$ and $25^{\text {th }}$ April in the first and second seasons, respectively. The preceding summer crop was the earth boor and the second season was sorghum in both seasons. All other practices were uniformly applied as recommended for wheat production in the region.

Table (1): Mechanical, chemical and physical properties of experimental site before the cultivation during 2017/2018 and 2018/2019 seasons, according to the methods described by Jackson (1975).

\begin{tabular}{|c|c|c|c|c|c|c|c|c|c|c|}
\hline \multicolumn{11}{|c|}{ Season $2017 / 2018$} \\
\hline \multirow{2}{*}{ EC } & \multirow{2}{*}{ PH } & \multirow{2}{*}{$\mathrm{CaCo}_{3}(\%)$} & \multicolumn{4}{|c|}{ Soluble Cation (meg/liter) } & \multicolumn{4}{|c|}{ Soluble anions (meg/liter) } \\
\hline & & & $\mathrm{Ca}$ & $\mathrm{Mg}$ & $\mathrm{Na}$ & 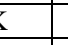 & \multicolumn{2}{|r|}{$\mathrm{HCO}_{3}$} & $\mathrm{Cl}$ & $\mathrm{So}_{4}$ \\
\hline 0.98 & 7.3 & 10.3 & 1.2 & 3.1 & 0.35 & 7 & 45 & 0.2 & 1.43 & 4.1 \\
\hline \multicolumn{5}{|c|}{ Macro nutrients (ppm) } & \multicolumn{6}{|c|}{ Micro nutrients (ppm) } \\
\hline \multicolumn{2}{|c|}{$\mathrm{N}$} & $\mathrm{P}$ & \multicolumn{2}{|c|}{$\mathrm{K}$} & \multicolumn{2}{|c|}{$\mathrm{Fe}$} & $\mathrm{Zn}$ & $\mathrm{Mn}$ & \multicolumn{2}{|c|}{$\mathrm{Cu}$} \\
\hline \multicolumn{2}{|c|}{20} & 4.8 & \multicolumn{2}{|c|}{292} & 5.5 & & 20 & 5.46 & 0.2 & \\
\hline \multicolumn{5}{|c|}{ Organic matter $(\%)$} & \multicolumn{6}{|c|}{ Soil texture } \\
\hline \multirow{2}{*}{\multicolumn{5}{|c|}{2.2}} & \multicolumn{3}{|c|}{ Sand $\%$} & \multicolumn{3}{|c|}{ Clay $(\%)$} \\
\hline & & & & & \multicolumn{3}{|c|}{12.12} & \multicolumn{3}{|c|}{87.88} \\
\hline \multicolumn{11}{|c|}{ Season $2018 / 2019$} \\
\hline \multirow{2}{*}{ EC } & \multirow{2}{*}{ PH } & \multirow{2}{*}{$\mathrm{CaCo}_{3}(\%)$} & \multicolumn{4}{|c|}{ Soluble Cation (meg/liter) } & & luble anic & $(\mathrm{meg} / \mathrm{li}$ & \\
\hline & & & $\mathrm{Ca}$ & $\mathrm{Mg}$ & $\mathrm{Na}$ & $\mathrm{K}$ & $\mathrm{Co}_{3}$ & $\mathrm{HCO}_{3}$ & $\mathrm{Cl}$ & So4 \\
\hline 1.06 & 8 & 13.8 & 1.4 & 3.4 & 0.39 & 1.10 & 0.47 & 0.2 & 1.47 & 4.6 \\
\hline & & cro nutrients ( & & & & & ro nutri & nts (ppm) & & \\
\hline & & $\mathrm{P}$ & & & & & $\mathrm{Zn}$ & $\mathrm{Mn}$ & & \\
\hline & & 5.3 & & & & & 0.31 & 5.88 & & \\
\hline & & rganic matter & & & & & Soil te & xture & & \\
\hline & & 38 & & & & and $\%$ & & & Clay\% & \\
\hline & & 5.0 & & & & 10.54 & & & 89.46 & \\
\hline
\end{tabular}

Table (2): Results of the moisture content of the soil.

\begin{tabular}{|l|c|c|}
\hline \multirow{2}{*}{ Season } & \multicolumn{2}{|c|}{ Moisture content (\%) } \\
\cline { 2 - 3 } & $2017 / 2018$ & $2018 / 2019$ \\
\hline 21 Day & 83.4 & 86.5 \\
\hline 28 Day & 79.1 & 81.2 \\
\hline 35 Day & 70.9 & 73.6 \\
\hline
\end{tabular}


Table (3): Mean of temperature data during two growing seasons.

\begin{tabular}{|l|c|c|c|c|}
\hline \multirow{2}{*}{ Months } & \multicolumn{4}{|c|}{ Mean of temperature $\left({ }^{\circ} \mathrm{C}\right)$} \\
\cline { 2 - 5 } & \multicolumn{2}{|c|}{$2017 / 2018$} & \multicolumn{2}{c|}{$2018 / 2019$} \\
\cline { 2 - 5 } & Max & Min & Max & Min \\
\hline November 15-30 & 28 & 13 & 29 & 13 \\
\hline December 1-15 & 26 & 11 & 23 & 10 \\
\hline December 16-31 & 27 & 12 & 22 & 8 \\
\hline January 1-15 & 22 & 8 & 19 & 6 \\
\hline January 16-31 & 23 & 7 & 22 & 7 \\
\hline February 1-15 & 27 & 10 & 24 & 9 \\
\hline February 16-28 & 29 & 12 & 25 & 11 \\
\hline March 1-15 & 33 & 15 & 26 & 10 \\
\hline March 16-31 & 37 & 18 & 29 & 13 \\
\hline April 1-15 & 37 & 18 & 32 & 14 \\
\hline April 16-30 & 36 & 19 & 33 & 16 \\
\hline
\end{tabular}

Source: Agricultural Meteorology Cell, Agricultural Directorate in Qena. https://ar.climatedata.org/location/3676. https://www.accuweather.com.

At harvest, ten plants were chosen randomly from each plot to measure the following characters:

1. 1000-grain weight $(\mathrm{g}): 1000$ tablets are taken and weighed.

2. Grains weight /spike (g).

3. Number of spikes $/ \mathrm{m}^{2}$ : $(1 \mathrm{~m} \times 1 \mathrm{~m}) \mathrm{m}^{2}$ Number of spikes in one square meter of each plot.

4. Grain yield (ardab/fed) $($ argab $=150$ $\mathrm{Kg}$, fed $=$ feddan $=42002 \mathrm{~m}^{2}=0.420$ hectares $=1.037$ acres): The grain of each plot $\left(10.5 \mathrm{~m}^{2}\right)$ was weighted and the mean grain yield (ardab/fed) was calculated.

5. Straw yield (ton/fed): determined by weighing the biological yield in each plot then substrating the grain weight for the whole plants, results were expressed as ton/fed.

6. Protein percentage (\%) in grain : Nitrogen determination was carried out by the improved Kieldhal method of A.O.A.C. (1980) which modified by distilling the ammonia into saturated boric solution and titration was carried out by using standard hydrochloric acid and calculated by using multiplying the total nitrogen in grain $\mathrm{x} 6.25$ (Protein percentage $=$ Grain $\mathrm{N} \% \times 6.25)$.

The recorded data were statistically analyzed in SAS software (SAS version 9.2, SAS Institute, 2008) according to Gomez and Gomez (1984), the least significant differences (LSD) at probability level at $0.05 \%$ was calculated according to Petersen (1985) for comparing the differences between treatment means of the studied traits.

\section{Results and Discussion}

Effect of planting methods and irrigation intervals on productivity of wheat cultivars on yield and yield components of three wheat cultivars in 2017/2018 and 2018/2019 seasons in Tables (4-9). Results showed the effect of wheat cultivars on 1000-grain weight (g), grains weight / spike $(\mathrm{g})$, number of spikes $/ \mathrm{m}^{2}$, grains yield ardab / fed, straw yield (ton/ 
fed) and protein percentage in grain (\%) were significant in both seasons. Sids-12 cultivars was gave the heaviest 1000grain (g) (45.21 and 52.26), grains weight / spike (g) (3.211 and 4.374), number of spikes/ $\mathrm{m}^{2}$ (291.1 and 309), grains yield (ardab / fed) (19.68 and 20.33) , straw yield (ton/ fed) (1.813 and $1.961)$ and protein percentage in grain
(\%) (11.31and 11.51) in the first seasons and second seasons, respectively. This result may be due to the interaction between genetic make-up and their environmental condition. Similar findings were found by EL-kalla et al. (2010), Badran (2011), Moustafa (2013), Abdelkhalek et al. (2015) and ElAshmouny et al (2016).

Table (4): 1000-grain weight (g)of some wheat varieties as affected by irrigation intervals and planting methods in 2017/2018 and 2018/2019 seasons.

\begin{tabular}{|c|c|c|c|c|c|c|c|c|c|}
\hline \multirow{5}{*}{$\begin{array}{l}\text { Irrigation } \\
\text { Intervals }\end{array}$} & \multirow{5}{*}{$\begin{array}{l}\text { Planting } \\
\text { Methods } \\
\text { (B) }\end{array}$} & \multicolumn{8}{|c|}{ Season } \\
\hline & & \multirow{2}{*}{\multicolumn{4}{|c|}{$\frac{2017 / 2018}{\text { Cultivars (V) }}$}} & \multicolumn{4}{|c|}{$2018 / 2019$} \\
\hline & & & & & & \multicolumn{4}{|c|}{ Cultivars (V) } \\
\hline & & Sids 12 & Giza 168 & Misr 2 & & Sids 12 & Giza 168 & Misr 2 & \\
\hline & & $\mathrm{V}_{1}$ & $\mathrm{~V}_{2}$ & $\mathrm{~V}_{3}$ & Mean & $\mathrm{V}_{1}$ & $V_{2}$ & $V_{3}$ & Mean \\
\hline \multirow{3}{*}{$\mathrm{I}_{1}$} & Drill $_{(\mathrm{B} 1)}$ & 56.23 & 52.50 & 53.47 & 54.07 & 62.83 & 59.57 & 61.20 & 61.20 \\
\hline & B. cast ${ }_{(\mathrm{B} 2)}$ & 45.90 & 37.27 & 38.57 & 40.58 & 54.47 & 52.33 & 53.93 & 53.58 \\
\hline & Furrows $_{(\mathrm{B} 3)}$ & 51.27 & 47.47 & 49.70 & 49.48 & 59.33 & 55.30 & 56.87 & 57.17 \\
\hline \multicolumn{2}{|l|}{ Mean } & 51.13 & 45.74 & 47.24 & 48.04 & 58.88 & 55.73 & 57.33 & 57.31 \\
\hline \multirow{3}{*}{$\mathrm{I}_{2}$} & B1 & 58.50 & 50.63 & 53.37 & 54.17 & 61.37 & 57.67 & 59.10 & 59.38 \\
\hline & B2 & 38.63 & 31.97 & 36.27 & 35.62 & 45.73 & 42.00 & 44.77 & 44.17 \\
\hline & B3 & 47.23 & 44.07 & 46.13 & 45.81 & 56.27 & 51.67 & 54.63 & 54.19 \\
\hline \multicolumn{2}{|l|}{ Mean } & 48.12 & 42.22 & 45.26 & 45.20 & 54.46 & 50.44 & 52.83 & 52.58 \\
\hline \multirow{3}{*}{$\mathrm{I}_{3}$} & B1 & 45.20 & 42.08 & 43.37 & 43.55 & 50.43 & 47.03 & 49.47 & 48.98 \\
\hline & $\mathrm{B} 2$ & 26.17 & 22.53 & 24.80 & 24.50 & 35.90 & 28.60 & 33.70 & 32.73 \\
\hline & B3 & 37.80 & 29.77 & 34.30 & 33.96 & 44.03 & 37.50 & 40.97 & 40.83 \\
\hline \multicolumn{2}{|l|}{ Mean } & 36.39 & 31.46 & 34.16 & 34.00 & 43.46 & 37.71 & 41.38 & 40.85 \\
\hline \multirow{3}{*}{$\begin{array}{l}\text { Mean for } \\
\text { Planting } \\
\text { methods }\end{array}$} & B1 & 53.31 & 36.90 & 45.43 & 45.21 & 58.21 & 45.37 & 53.21 & 52.26 \\
\hline & $\mathrm{B} 2$ & 48.40 & 30.59 & 40.43 & 39.81 & 54.76 & 40.98 & 48.16 & 47.97 \\
\hline & B3 & 50.07 & 33.21 & 43.38 & 42.22 & 56.59 & 44.13 & 50.82 & 50.51 \\
\hline \multicolumn{2}{|l|}{ Mean } & 45.21 & 39.81 & 42.22 & & 52.26 & 47.96 & 50.51 & \\
\hline \multicolumn{10}{|c|}{ L.S.D. at $5 \%$ for : } \\
\hline \multicolumn{2}{|c|}{ Cultivars (V) } & \multicolumn{4}{|c|}{1.29} & \multicolumn{4}{|c|}{1.25} \\
\hline \multicolumn{2}{|c|}{ Irrigation ( I ) } & \multicolumn{4}{|c|}{0.6} & \multicolumn{4}{|c|}{1.14} \\
\hline \multicolumn{2}{|c|}{ Planting methods (B) } & \multicolumn{4}{|c|}{0.63} & \multicolumn{4}{|c|}{0.49} \\
\hline \multicolumn{2}{|l|}{ V X I } & \multicolumn{4}{|c|}{1.04} & \multicolumn{4}{|c|}{1.97} \\
\hline \multicolumn{2}{|l|}{ VX B } & \multicolumn{4}{|c|}{-} & & 0.8 & & \\
\hline I X B & & & - & & & & 0.8 & & \\
\hline V X I X B & & & 1.8 & & & & - & & \\
\hline
\end{tabular}

$\mathrm{V}=$ Cultivars, $\mathrm{B}=$ planting methods, $\mathrm{I}=$ irrigation, $\mathrm{V}_{1}=$ variety sids $12, \mathrm{~B}_{1}=$ Drill, $\mathrm{I}_{1}=21$ day, $\mathrm{V}_{2}=$ variety Giza 168 , $B_{2}=B$. cast, $I_{2}=28$ day, $V_{3}=$ variety Misr $2, B_{3}=$ furrows, $I_{3}=35$ day.

Results showed that the effect of irrigation intervals on all characteristics studied was significant in both seasons. The highest values of all the characteristics studied were found from the 21 days during growing season followed by the 28 days withe significant differences. The lowest values for all characteristics studied were obtained from 35 days in both seasons. 
Table (5): Grains weight/spike (g) of some wheat varieties as affected by irrigation intervals and planting methods in 2017/2018 and 2018/2019 seasons.

\begin{tabular}{|c|c|c|c|c|c|c|c|c|c|}
\hline \multirow{5}{*}{$\begin{array}{l}\text { Irrigation } \\
\text { Intervals }\end{array}$} & \multirow{5}{*}{$\begin{array}{l}\text { Planting } \\
\text { Methods } \\
\text { (B) }\end{array}$} & \multicolumn{8}{|c|}{ Season } \\
\hline & & \multicolumn{4}{|c|}{$2017 / 2018$} & \multicolumn{4}{|c|}{$2018 / 2019$} \\
\hline & & \multicolumn{4}{|c|}{ Cultivars (V) } & \multicolumn{4}{|c|}{ Cultivars (V) } \\
\hline & & Sids 12 & Giza 168 & Misr 2 & & Sids 12 & Giza 168 & Misr 2 & \\
\hline & & $\mathrm{V}_{1}$ & $\mathrm{~V}_{2}$ & $\mathrm{~V}_{3}$ & Mean & $\mathrm{V}_{1}$ & $\mathrm{~V}_{2}$ & $\mathrm{~V}_{3}$ & Mean \\
\hline \multirow{3}{*}{$\mathrm{I}_{1}$} & Drill $_{(\mathrm{Bl})}$ & 4.067 & 3.733 & 3.900 & 3.900 & 5.067 & 3.867 & 4.667 & 4.534 \\
\hline & B. cast $_{(\mathrm{B} 2)}$ & 3.133 & 2.833 & 3.033 & 3.000 & 4.500 & 3.700 & 4.067 & 4.089 \\
\hline & Furrows $_{(\mathrm{B} 3)}$ & 3.567 & 3.333 & 3.467 & 3.456 & 4.533 & 3.700 & 4.233 & 4.155 \\
\hline \multicolumn{2}{|l|}{ Mean } & 3.590 & 3.300 & 3.470 & 3.453 & 4.700 & 3.760 & 4.320 & 4.260 \\
\hline \multirow{3}{*}{$\mathrm{I}_{2}$} & B1 & 3.733 & 3.300 & 3.533 & 3.522 & 4.800 & 3.667 & 4.467 & 4.311 \\
\hline & $\mathrm{B} 2$ & 2.567 & 2.400 & 2.733 & 2.567 & 4.167 & 3.367 & 3.767 & 3.767 \\
\hline & B3 & 3.200 & 2.900 & 3.033 & 3.044 & 4.333 & 3.467 & 3.967 & 3.922 \\
\hline \multicolumn{2}{|l|}{ Mean } & 3.170 & 2.870 & 3.100 & 3.047 & 4.430 & 3.500 & 4.070 & 4.000 \\
\hline \multirow{3}{*}{$\mathrm{I}_{3}$} & B1 & 3.600 & 2.967 & 3.167 & 3.245 & 4.400 & 3.433 & 4.033 & 3.955 \\
\hline & $\mathrm{B} 2$ & 2.300 & 1.967 & 2.167 & 2.145 & 3.733 & 2.733 & 3.267 & 3.244 \\
\hline & B3 & 2.733 & 2.433 & 2.567 & 2.578 & 3.833 & 3.200 & 3.633 & 3.555 \\
\hline \multicolumn{2}{|l|}{ Mean } & 2.880 & 2.460 & 2.630 & 2.657 & 3.990 & 3.120 & 3.640 & 3.583 \\
\hline \multirow{3}{*}{$\begin{array}{l}\text { Mean for } \\
\text { Planting } \\
\text { methods }\end{array}$} & $\mathrm{B} 1$ & 3.800 & 2.667 & 3.167 & 3.211 & 4.756 & 4.133 & 4.233 & 4.374 \\
\hline & $\mathrm{B} 2$ & 3.333 & 2.400 & 2.889 & 2.874 & 3.656 & 3.267 & 3.456 & 3.460 \\
\hline & B3 & 3.533 & 2.644 & 3.022 & 3.066 & 4.389 & 3.700 & 3.944 & 4.011 \\
\hline \multicolumn{2}{|l|}{ Mean } & 3.211 & 2.874 & 3.067 & & 4.374 & 3.459 & 4.011 & \\
\hline \multicolumn{10}{|c|}{ L.S.D. at $5 \%$ for : } \\
\hline \multicolumn{2}{|c|}{ Cultivars (V) } & \multicolumn{4}{|c|}{0.13} & \multicolumn{4}{|c|}{0.1} \\
\hline \multicolumn{2}{|c|}{ Irrigation ( I ) } & \multicolumn{4}{|c|}{0.07} & \multicolumn{4}{|c|}{0.08} \\
\hline \multicolumn{2}{|c|}{ Planting methods (B) } & \multicolumn{4}{|c|}{0.06} & \multicolumn{4}{|c|}{0.1} \\
\hline \multicolumn{2}{|c|}{ V X I } & \multicolumn{4}{|c|}{-} & \multicolumn{4}{|c|}{-} \\
\hline \multicolumn{2}{|l|}{ V X B } & \multicolumn{4}{|c|}{-} & & - & & \\
\hline IX B & & & 0 . & & & & 0. & & \\
\hline VXIX B & & & 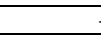 & & & & 0. & & \\
\hline
\end{tabular}

$\mathrm{V}=$ Cultivars, $\mathrm{B}=$ planting methods, $\mathrm{I}=$ irrigation, $\mathrm{V}_{1}=$ variety sids $12, \mathrm{~B}_{1}=$ Drill, $\mathrm{I}_{1}=21$ day, $\mathrm{V}_{2}=$ variety Giza $168, \mathrm{~B}_{2}=\mathrm{B}$. cast, $\mathrm{I}_{2}=28$ day, $\mathrm{V}_{3}=$ variety Misr 2, $\mathrm{B}_{3}=$ furrows, $\mathrm{I}_{3}=35$ day.

Table (6): Number of spikes /m2of some wheat varieties as affected by irrigation intervals and planting methods in 2017/2018 and 2018/2019 seasons.

\begin{tabular}{|c|c|c|c|c|c|c|c|c|c|}
\hline \multirow{5}{*}{$\begin{array}{l}\text { Irrigation } \\
\text { Intervals }\end{array}$} & \multirow{5}{*}{$\begin{array}{l}\text { Planting } \\
\text { Methods } \\
\text { (B) }\end{array}$} & \multicolumn{8}{|c|}{ Season } \\
\hline & & \multicolumn{4}{|c|}{$2017 / 2018$} & \multicolumn{4}{|c|}{$2018 / 2019$} \\
\hline & & \multicolumn{4}{|c|}{ Cultivars (V) } & \multicolumn{4}{|c|}{ Cultivars (V) } \\
\hline & & Sids 12 & Giza 168 & Misr 2 & & Sids 12 & Giza 168 & Misr 2 & \\
\hline & & $\mathrm{V}_{1}$ & $\mathrm{~V}_{2}$ & $\mathrm{~V}_{3}$ & Mean & $\mathrm{V}_{1}$ & $\mathrm{~V}_{2}$ & $\mathrm{~V}_{3}$ & Mean \\
\hline \multirow{3}{*}{$\mathrm{I}_{1}$} & $\operatorname{Drill}_{(\mathrm{B} 1)}$ & 375.3 & 346.3 & 362.3 & 361.3 & 379.7 & 349.7 & 365.0 & 364.8 \\
\hline & B. cast $_{(\mathrm{B} 2)}$ & 260.7 & 244.3 & 249.0 & 251.3 & 295.7 & 272.7 & 288.0 & 285.5 \\
\hline & Furrows $_{(\mathrm{B} 3)}$ & 292.0 & 264.7 & 279.3 & 278.7 & 321.3 & 285.7 & 308.3 & 305.1 \\
\hline \multicolumn{2}{|l|}{ Mean } & 309.3 & 285.1 & 296.9 & 297.1 & 332.2 & 302.7 & 320.4 & 318.4 \\
\hline \multirow{3}{*}{$\mathrm{I}_{2}$} & $\mathrm{~B} 1$ & 371.0 & 325.3 & 346.0 & 347.4 & 377.7 & 337.3 & 359.3 & 358.1 \\
\hline & $\mathrm{B} 2$ & 257.0 & 238.0 & 239.7 & 244.9 & 273.0 & 254.0 & 264.3 & 263.8 \\
\hline & B3 & 271.0 & 240.3 & 249.3 & 253.5 & 319.0 & 280.7 & 304.3 & 301.3 \\
\hline \multicolumn{2}{|l|}{ Mean } & 299.7 & 267.9 & 278.3 & 282.0 & 323.2 & 290.7 & 309.3 & 307.7 \\
\hline \multirow{3}{*}{$\mathrm{I}_{3}$} & B1 & 310.3 & 268.3 & 282.0 & 286.9 & 316.7 & 278.7 & 300.0 & 298.5 \\
\hline & $\mathrm{B} 2$ & 235.3 & 226.7 & 230.3 & 230.8 & 247.3 & 230.0 & 236.3 & 237.9 \\
\hline & B3 & 247.7 & 232.7 & 241.3 & 240.6 & 250.7 & 238.7 & 247.0 & 245.5 \\
\hline \multicolumn{2}{|l|}{ Mean } & 264.4 & 242.6 & 251.2 & 252.7 & 271.6 & 249.1 & 261.1 & 260.6 \\
\hline \multirow{3}{*}{$\begin{array}{l}\text { Mean for } \\
\text { Planting } \\
\text { methods }\end{array}$} & $\mathrm{B} 1$ & 352.2 & 251.0 & 270.2 & 291.1 & 358.0 & 272.0 & 297.0 & 309.0 \\
\hline & $\mathrm{B} 2$ & 313.3 & 236.3 & 245.9 & 265.2 & 321.9 & 252.2 & 268.3 & 280.8 \\
\hline & B3 & 330.1 & 239.7 & 256.7 & 275.5 & 341.4 & 262.9 & 286.6 & 297.0 \\
\hline \multicolumn{2}{|l|}{ Mean } & 291.1 & 265.2 & 275.5 & & 309.0 & 280.8 & 297.0 & \\
\hline \multicolumn{10}{|c|}{ L.S.D. at $5 \%$ for : } \\
\hline \multicolumn{2}{|c|}{ Cultivars (V) } & \multicolumn{4}{|c|}{2.98} & \multicolumn{4}{|c|}{3.86} \\
\hline \multicolumn{2}{|c|}{ Irrigation ( I ) } & \multicolumn{4}{|c|}{4.05} & \multicolumn{4}{|c|}{3.55} \\
\hline \multicolumn{2}{|c|}{ Planting methods (B) } & \multicolumn{4}{|c|}{2.44} & \multicolumn{4}{|c|}{2.66} \\
\hline \multicolumn{2}{|c|}{ VX I } & \multicolumn{4}{|c|}{7.01} & \multicolumn{4}{|c|}{6.15} \\
\hline \multicolumn{2}{|l|}{ V X B } & \multicolumn{4}{|c|}{4.22} & & 4. & & \\
\hline I X B & & & 4. & & & & 4. & & \\
\hline V X IX B & & & 7. & & & & 7. & & \\
\hline
\end{tabular}

$\mathrm{V}=$ Cultivars, $\mathrm{B}=$ planting methods, $\mathrm{I}=$ irrigation, $\mathrm{V}_{1}=$ variety sids $12, \mathrm{~B}_{1}=$ Drill, $\mathrm{I}_{1}=21$ day, $\mathrm{V}_{2}=$ variety Giza $168, \mathrm{~B}_{2}=\mathrm{B}$. cast, $\mathrm{I}_{2}=28$ day, $\mathrm{V}_{3}=$ variety Misr $2, \mathrm{~B}_{3}=$ furrows, $\mathrm{I}_{3}=35$ day. 
Table (7): Grains yield (ardab/fed) of some wheat varieties as affected by irrigation intervals and planting methods in 2017/2018 and 2018/2019 seasons.

\begin{tabular}{|c|c|c|c|c|c|c|c|c|c|}
\hline \multirow{5}{*}{$\begin{array}{l}\text { Irrigation } \\
\text { Intervals }\end{array}$} & \multirow{5}{*}{$\begin{array}{l}\text { Planting } \\
\text { Methods } \\
\text { (B) }\end{array}$} & \multicolumn{8}{|c|}{ Season } \\
\hline & & \multicolumn{4}{|c|}{$2017 / 2018$} & \multicolumn{4}{|c|}{$2018 / 2019$} \\
\hline & & \multicolumn{4}{|c|}{ Cultivars (V) } & \multicolumn{4}{|c|}{ Cultivars (V) } \\
\hline & & Sids 12 & Giza 168 & Misr 2 & & Sids 12 & Giza 168 & Misr 2 & \\
\hline & & $\mathrm{V}_{1}$ & $\mathrm{~V}_{2}$ & $\mathrm{~V}_{3}$ & Mean & $\mathrm{V}_{1}$ & $\mathrm{~V}_{2}$ & $\mathrm{~V}_{3}$ & Mean \\
\hline \multirow{3}{*}{$\mathrm{I}_{1}$} & \begin{tabular}{|l} 
Drill $_{(\mathrm{Bl})}$ \\
\end{tabular} & 22.27 & 20.22 & 21.69 & 21.39 & 23.33 & 20.95 & 21.87 & 22.05 \\
\hline & B. cast $_{(\mathrm{B} 2)}$ & 17.67 & 16.19 & 16.96 & 16.94 & 18.09 & 16.57 & 17.33 & 17.33 \\
\hline & Furrows $_{(\mathrm{B} 3)}$ & 21.87 & 18.87 & 19.57 & 20.10 & 22.14 & 19.43 & 20.69 & 20.75 \\
\hline \multicolumn{2}{|l|}{ Mean } & 20.60 & 18.43 & 19.42 & 19.48 & 21.19 & 18.98 & 19.96 & 20.04 \\
\hline \multirow{3}{*}{$\mathrm{I}_{2}$} & $\mathrm{~B} 1$ & 21.27 & 19.24 & 20.14 & 20.22 & 22.57 & 20.14 & 21.55 & 21.42 \\
\hline & $\mathrm{B} 2$ & 16.79 & 15.87 & 16.43 & 16.36 & 17.16 & 16.20 & 17.12 & 16.82 \\
\hline & B3 & 21.34 & 18.04 & 18.87 & 19.42 & 21.80 & 18.55 & 20.21 & 20.19 \\
\hline \multicolumn{2}{|l|}{ Mean } & 19.80 & 17.72 & 18.48 & 18.67 & 20.51 & 18.30 & 19.63 & 19.48 \\
\hline \multirow{3}{*}{$\mathrm{I}_{3}$} & B1 & 20.37 & 18.90 & 20.12 & 19.76 & 21.00 & 19.86 & 20.45 & 20.44 \\
\hline & $\mathrm{B} 2$ & 15.85 & 14.08 & 14.33 & 14.76 & 16.68 & 14.35 & 16.08 & 15.73 \\
\hline & B3 & 19.69 & 16.91 & 17.97 & 18.19 & 20.23 & 17.84 & 18.36 & 18.81 \\
\hline \multicolumn{2}{|l|}{ Mean } & 18.64 & 16.63 & 17.47 & 17.57 & 19.33 & 17.35 & 18.30 & 18.33 \\
\hline \multirow{3}{*}{$\begin{array}{l}\text { Mean for } \\
\text { Planting } \\
\text { methods }\end{array}$} & $\mathrm{B} 1$ & 21.30 & 19.45 & 20.65 & 20.46 & 22.30 & 20.32 & 21.29 & 21.30 \\
\hline & $\mathrm{B} 2$ & 16.77 & 15.38 & 15.91 & 16.02 & 17.31 & 15.71 & 16.84 & 16.63 \\
\hline & B3 & 20.97 & 17.94 & 18.80 & 19.24 & 21.39 & 18.61 & 19.75 & 19.92 \\
\hline \multicolumn{2}{|l|}{ Mean } & 19.68 & 17.59 & 18.45 & & 20.33 & 18.21 & 19.30 & \\
\hline \multicolumn{10}{|c|}{ L.S.D. at $5 \%$ for : } \\
\hline \multicolumn{2}{|c|}{ Cultivars (V) } & \multicolumn{4}{|c|}{1.76} & \multicolumn{4}{|c|}{1.60} \\
\hline \multicolumn{2}{|c|}{ Irrigation ( I ) } & \multicolumn{4}{|c|}{1.97} & \multicolumn{4}{|c|}{1.91} \\
\hline \multicolumn{2}{|c|}{ Planting methods (B) } & \multicolumn{4}{|c|}{1.38} & \multicolumn{4}{|c|}{1.57} \\
\hline \multicolumn{2}{|l|}{ V X I } & \multicolumn{4}{|c|}{1.53} & \multicolumn{4}{|c|}{1.24} \\
\hline \multicolumn{2}{|l|}{ VX B } & \multicolumn{4}{|c|}{1.82} & & 1. & & \\
\hline IX B & & & & & & & 1. & & \\
\hline V X I X B & & & & & & & 1. & & \\
\hline
\end{tabular}

$\mathrm{V}=$ Cultivars, $\mathrm{B}=$ planting methods, $\mathrm{I}=$ irrigation, $\mathrm{V}_{1}=$ variety sids $12, \mathrm{~B}_{1}=$ Drill, $\mathrm{I}_{1}=21$ day, $\mathrm{V}_{2}=$ variety Giza $168, \mathrm{~B}_{2}=\mathrm{B}$. cast, $\mathrm{I}_{2}=28$ day, $\mathrm{V}_{3}=$ variety Misr 2, $\mathrm{B}_{3}=$ furrows, $\mathrm{I}_{3}=35$ day.

Table (8): Straw yield (ton/fed) of some wheat varieties as affected by irrigation intervals and planting methods in 2017/2018 and 2018/2019 seasons.

\begin{tabular}{|c|c|c|c|c|c|c|c|c|c|}
\hline \multirow{5}{*}{$\begin{array}{l}\text { Irrigation } \\
\text { Intervals }\end{array}$} & \multirow{5}{*}{$\begin{array}{l}\text { Planting } \\
\text { Methods } \\
\text { (B) }\end{array}$} & \multicolumn{8}{|c|}{ Season } \\
\hline & & \multicolumn{4}{|c|}{$2017 / 2018$} & \multicolumn{4}{|c|}{$2018 / 2019$} \\
\hline & & \multicolumn{4}{|c|}{ Cultivars (V) } & \multicolumn{4}{|c|}{ Cultivars (V) } \\
\hline & & Sids 12 & Giza 168 & Misr 2 & & Sids 12 & Giza 168 & Misr 2 & \\
\hline & & $\mathrm{V}_{1}$ & $\mathrm{~V}_{2}$ & $\mathrm{~V}_{3}$ & Mean & $\mathrm{V}_{1}$ & $\mathrm{~V}_{2}$ & $\mathrm{~V}_{3}$ & Mean \\
\hline \multirow{3}{*}{$\mathrm{I}_{1}$} & Drill $_{(\mathrm{B} 1)}$ & 1.808 & 1.950 & 1.843 & 1.867 & 2.103 & 1.738 & 1.708 & 1.850 \\
\hline & B. cast $_{(\mathrm{B} 2)}$ & 1.928 & 1.985 & 1.948 & 1.954 & 1.927 & 1.978 & 1.933 & 1.946 \\
\hline & Furrows $_{(\mathrm{B} 3)}$ & 1.985 & 1.937 & 2.090 & 2.004 & 2.000 & 2.058 & 2.137 & 2.065 \\
\hline \multicolumn{2}{|l|}{ Mean } & 1.910 & 1.960 & 1.960 & 1.943 & 2.010 & 1.920 & 1.930 & 1.953 \\
\hline \multirow{3}{*}{$\mathrm{I}_{2}$} & $\mathrm{~B} 1$ & 1.893 & 1.890 & 1.832 & 1.872 & 2.152 & 1.967 & 1.797 & 1.972 \\
\hline & $\mathrm{B} 2$ & 1.860 & 1.507 & 1.667 & 1.678 & 1.917 & 1.734 & 1.817 & 1.823 \\
\hline & B3 & 1.743 & 1.835 & 1.837 & 1.805 & 2.038 & 1.912 & 1.983 & 1.978 \\
\hline \multicolumn{2}{|l|}{ Mean } & 1.830 & 1.740 & 1.780 & 1.783 & 2.040 & 1.870 & 1.870 & 1.927 \\
\hline \multirow{3}{*}{$\mathrm{I}_{3}$} & $\mathrm{~B} 1$ & 1.817 & 1.762 & 1.710 & 1.763 & 1.955 & 1.942 & 1.653 & 1.850 \\
\hline & $\mathrm{B} 2$ & 1.637 & 1.477 & 1.662 & 1.592 & 1.692 & 1.790 & 1.713 & 1.732 \\
\hline & B3 & 1.642 & 1.652 & 1.797 & 1.697 & 1.867 & 1.787 & 2.003 & 1.886 \\
\hline \multicolumn{2}{|l|}{ Mean } & 1.700 & 1.630 & 1.720 & 1.683 & 1.840 & 1.840 & 1.790 & 1.823 \\
\hline \multirow{3}{*}{$\begin{array}{l}\text { Mean for } \\
\text { Planting } \\
\text { methods }\end{array}$} & $\mathrm{B} 1$ & 1.839 & 1.808 & 1.790 & 1.821 & 2.070 & 1.845 & 1.968 & 1.961 \\
\hline & $\mathrm{B} 2$ & 1.867 & 1.656 & 1.808 & 1.777 & 1.882 & 1.834 & 1.919 & 1.878 \\
\hline & B3 & 1.795 & 1.759 & 1.908 & 1.812 & 1.719 & 1.821 & 2.041 & 1.860 \\
\hline \multicolumn{2}{|l|}{ Mean } & 1.813 & 1.777 & 1.821 & & 1.961 & 1.878 & 1.861 & \\
\hline \multicolumn{10}{|c|}{ L.S.D. at $5 \%$ for : } \\
\hline \multicolumn{2}{|c|}{ Cultivars (V) } & \multicolumn{4}{|c|}{0.05} & \multicolumn{4}{|c|}{0.07} \\
\hline \multicolumn{2}{|c|}{ Irrigation ( I ) } & \multicolumn{4}{|c|}{0.04} & \multicolumn{4}{|c|}{0.07} \\
\hline \multicolumn{2}{|c|}{ Planting methods (B) } & \multicolumn{4}{|c|}{0.05} & \multicolumn{4}{|c|}{0.05} \\
\hline \multicolumn{2}{|c|}{ V X I } & \multicolumn{4}{|c|}{0.08} & \multicolumn{4}{|c|}{0.12} \\
\hline \multicolumn{2}{|l|}{ V X B } & \multicolumn{4}{|c|}{-} & & . & & \\
\hline IX B & & & 0. & & & & 0. & & \\
\hline V X I X B & & & 0. & & & & 0. & & \\
\hline
\end{tabular}

$\mathrm{V}=$ Cultivars, $\mathrm{B}=$ planting methods, $\mathrm{I}=$ irrigation, $\mathrm{V}_{1}=$ variety sids $12, \mathrm{~B}_{1}=$ Drill, $\mathrm{I}_{1}=21$ day, $\mathrm{V}_{2}=$ variety Giza $168, \mathrm{~B}_{2}=\mathrm{B}$. cast, $\mathrm{I}_{2}=28$ day, $\mathrm{V}_{3}=$ variety Misr $2, \mathrm{~B}_{3}=$ furrows, $\mathrm{I}_{3}=35$ day. 
Table (9): Protein percentage in grain (\%) of some wheat varieties as affected by irrigation intervals and planting methods in 2017/2018 and 2018/2019 seasons.

\begin{tabular}{|c|c|c|c|c|c|c|c|c|c|}
\hline \multirow{5}{*}{$\begin{array}{l}\text { Irrigation } \\
\text { Intervals }\end{array}$} & \multirow{5}{*}{$\begin{array}{l}\text { Planting } \\
\text { Methods } \\
\text { (B) }\end{array}$} & \multicolumn{8}{|c|}{ Season } \\
\hline & & \multicolumn{4}{|c|}{$2017 / 2018$} & \multicolumn{4}{|c|}{$2018 / 2019$} \\
\hline & & \multicolumn{4}{|c|}{ Cultivars (V) } & \multicolumn{4}{|c|}{ Cultivars (V) } \\
\hline & & Sids 12 & Giza 168 & Misr 2 & & Sids 12 & Giza 168 & Misr 2 & \\
\hline & & $\mathrm{V}_{1}$ & $\mathrm{~V}_{2}$ & $\mathrm{~V}_{3}$ & Mean & $\mathrm{V}_{1}$ & $\mathrm{~V}_{2}$ & $\mathrm{~V}_{3}$ & Mean \\
\hline \multirow{3}{*}{$\mathrm{I}_{1}$} & Drill $_{(\mathrm{Bl})}$ & 12.48 & 7.913 & 9.993 & 10.13 & 12.68 & 9.163 & 11.25 & 11.03 \\
\hline & B. cast ${ }_{(\mathrm{B} 2)}$ & 11.66 & 7.480 & 8.707 & 9.283 & 11.52 & 6.893 & 8.937 & 9.117 \\
\hline & \begin{tabular}{|l|} 
Furrows $_{(\mathrm{B} 3)}$ \\
\end{tabular} & 11.92 & 7.667 & 9.583 & 9.722 & 12.31 & 8.207 & 9.787 & 10.10 \\
\hline \multicolumn{2}{|l|}{ Mean } & 12.02 & 7.687 & 9.428 & 9.711 & 12.17 & 8.088 & 9.990 & 10.09 \\
\hline \multirow{3}{*}{$\mathrm{I}_{2}$} & \begin{tabular}{|l|} 
B1 \\
\end{tabular} & 11.92 & 6.873 & 9.373 & 9.386 & 12.50 & 7.813 & 9.997 & 10.11 \\
\hline & \begin{tabular}{|l|}
$\mathrm{B} 2$ \\
\end{tabular} & 10.88 & 6.873 & 7.850 & 8.532 & 11.02 & 7.540 & 8.623 & 9.060 \\
\hline & B3 & 11.39 & 7.063 & 7.497 & 8.651 & 11.44 & 7.667 & 8.373 & 9.160 \\
\hline \multicolumn{2}{|l|}{ Mean } & 11.39 & 6.937 & 8.240 & 8.857 & 11.65 & 7.673 & 8.998 & 9.441 \\
\hline \multirow{3}{*}{$\mathrm{I}_{3}$} & B1 & 11.11 & 5.937 & 8.747 & 8.596 & 11.50 & 6.937 & 8.747 & 9.060 \\
\hline & \begin{tabular}{|l|} 
B2 \\
\end{tabular} & 9.997 & 5.813 & 7.437 & 7.749 & 10.11 & 6.540 & 7.417 & 8.020 \\
\hline & \begin{tabular}{|l|} 
B3 \\
\end{tabular} & 10.42 & 5.623 & 6.457 & 7.498 & 10.50 & 6.020 & 7.290 & 7.936 \\
\hline \multicolumn{2}{|l|}{ Mean } & 10.51 & 5.791 & 7.547 & 7.947 & 10.71 & 6.499 & 7.818 & 8.339 \\
\hline \multirow{3}{*}{$\begin{array}{l}\text { Mean for } \\
\text { Planting } \\
\text { methods }\end{array}$} & B1 & 11.83 & 6.908 & 9.371 & 9.370 & 12.23 & 7.971 & 9.997 & 10.07 \\
\hline & \begin{tabular}{|l|} 
B2 \\
\end{tabular} & 10.85 & 6.722 & 7.998 & 8.521 & 10.88 & 6.991 & 8.326 & 8.732 \\
\hline & B3 & 11.24 & 6.784 & 7.846 & 8.624 & 11.42 & 7.298 & 8.483 & 9.066 \\
\hline \multicolumn{2}{|l|}{ Mean } & 11.31 & 6.805 & 8.405 & & 11.51 & 7.420 & 8.935 & \\
\hline \multicolumn{10}{|c|}{ L.S.D. at $5 \%$ for : } \\
\hline \multicolumn{2}{|c|}{ Cultivars (V) } & \multicolumn{4}{|c|}{0.339} & \multicolumn{4}{|c|}{0.325} \\
\hline \multicolumn{2}{|c|}{ Irrigation ( I ) } & \multicolumn{4}{|c|}{0.178} & \multicolumn{4}{|c|}{0.295} \\
\hline \multicolumn{2}{|c|}{ Planting methods (B) } & \multicolumn{4}{|c|}{0.293} & \multicolumn{4}{|c|}{0.289} \\
\hline \multicolumn{2}{|c|}{ V X I } & \multicolumn{4}{|c|}{0.309} & \multicolumn{4}{|c|}{0.512} \\
\hline \multicolumn{2}{|l|}{ V X B } & \multicolumn{4}{|c|}{-} & & - & & \\
\hline IX B & & & 0.5 & & & & - & & \\
\hline VXIX B & & & 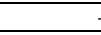 & & & & - & & \\
\hline
\end{tabular}

This may be due to water stress during grain filling stages, induced early senescence, reduced photosynthesis, also, effected on the transferring of carbohydrates from vegetative tissues to the grain and shortened grain filling period. These results are agreement with those obtained by Alsayim et al. (2013), Abdelkhalek et al. (2015), Seleiman and Abdel-Aal (2018), El-Metwally and Gad (2019) and Mohammed et al. (2019). Results showed that the effect of planting methods on all characteristics studied was significant in both seasons. Drilling method was gave the heaviest 1000- grain weight (g) (45.21 and 52.26), grains weight / spike (g) (3.211 and 4.374), number of spikes/ $\mathrm{m}^{2}$ (291.1 and 309), grains yield ardab/fed (20.65 and 21.30), straw yield ton/ fed (1.821 and 1.961) and protein percentage in grain (\%) $(9.370$ and 10.07) in the first seasons and second seasons, respectively. These results could be to the drilling methods had led to the optimum conditions of good standing, better field air circulation and such effects increased plant vegetative growth. These results are in harmony with those obtained by Ismail et al. (2008), ElAshmouny et al. (2011), Mahmoud et al. (2016), Hefny et al. (2015), El-Sherif et al. (2016) and Na-Allah et al. (2018). The interactions between cultivars and irrigation intervals were significant in both seasons for all characteristics studied, except grains weight/spike (g) in the both seasons. The interactions between cultivars and planting methods was significant in both seasons for all characteristics studied, except 1000- grain 
weight $(\mathrm{g})$ in the second season and grains weight/spike (g), straw yield ton/fed and protein percentage in grain $(\%)$ in the both seasons, respectively. The interactions between irrigation intervals and planting methods were significant in the first and second season for all characteristics studied, except 1000- grain weight $(\mathrm{g})$ in the second season and protein percentage in grain $(\%)$ in the first season only. Interaction effect of cultivars $\mathrm{x}$ irrigation treatment $\mathrm{x}$ planting methods was significant for all studied traits in the both seasons, except 1000- grain weight (g) in the first season and grains weight / spike $(\mathrm{g})$ in the second season. One of the most important factors that affect wheat productivity is planting methods and irrigation intervals. Our results showed exposing wheat plants to drought stress by increasing irrigation intervals significantly decrease (1000-grain weight, grain weight/spike, number of spikes/ $\mathrm{m}^{2}$, grain yield (ardab/ fed), straw yield (ton/fed) and protein (\%). Irrigation intervals showed highly significant difference in for all characters study. The highest value resulted from application Irrigation intervals at 21 day compare with the other irrigation intervals planting methods significantly affected on all a previous characters. The highest value on all study characters resulted from using drilling method. There were highly significant differences among wheat cultivars in for all characters under study.

\section{References}

Abdelkhalek, A. A., Darwesh, R. Kh. and El-Mansoury, A. M. (2015), "Response of some wheat varieties to irrigation and nitrogen fertilization using ammonia gas in North Nile Delta region", Annals of Agricultural Science, Vol. 60 No. 2, pp. 245-256.

Alsayim, E. H., El-Mahdi, A. A. and Naye, M. H. (2013), "Water- use efficiency of two wheat cultivars (Triticum aestivum L.) under tropical high terrace soil conditions", Asian Journal of Agriculture and Food Science, Vol. 1 No. 5, pp. 210-216.

Attia, A .N. E., Seadh, S. E., Sharshar, M. S. E. and Genedy, M. S. (2013), "Comparative studies on number of irrigations, planting methods and nitrogen levels for wheat in North Delta soils", Mansoura Journal of Plant Production, Vol. 4 No.7, pp. 1139-1148.

Abdelghany,A. M; H. M. Abouzied and M.S. Badran (2014), "Evaluation of some Egyptian wheat cultivars under water stress condition in the north western coast of Egypt", Journal of Plant Production, Vol. 12 No. 6, pp. $1-13$.

Braune, H. J., Atlin, G. and Payne, T. (2010), "Multi-location testing as a tool to identify plant response to global climate change", In: Climate change and crop production, Reynolds M. P. (Ed), CABI International, pp 115-138.

Badran, M. S. S. (2011), "Effect of soil water stress and deficit mineral nitrogen fertilization on productivity 
of inoculated wheat seeds with Bio$\mathrm{n}$ fertilizer cereal in sandy soil", Journal of Agricultural and Environmental Sciences, Vol. 10 No. 2, pp. 61-91.

El- Ashmouny M. S; Tantawy, A. A., Salem, M. A. and Hussien, O. M. (2016), "Effect of sowing and weed control methods on yield and its components of some bread wheat cultivars", El-Minia Journal of Agricultural Research and Development, Vol. 36 No. 4, pp. 551-563.

El-Ashmouny, M. S., Salam, M. A. and Ahmed, A. H. (2011), "Effect of sowing methods and seeding rates on yield and its components of some wheat cultivars", El-Minia Journal of Agricultural Research and Development, Vol. 31(3): 403-419.

El-Gabry, Y. A. and Hashem, F. A. (2018), "Yield and water productivity of bread wheat cultivars under diversified irrigation regimes", Journal of Nature and Science, Vol. 16 No. 1, pp. 143-149.

El-kalla, S. E., Leillah, A. A., El-Emery, M. I. and Kishk, A. M. S. (2010), "Performance of some wheat (Tritcum aestivum) cultivars under late sowing in newly reclaimed soils", Mansoura Journal of Plant Production, Vol. 1 No. 5, pp. 689697.

El-Metwally, I. M and Gad, N. (2019), "Wheat productivity and water use efficiency responses to irrigation, cobalt and weed management", Italian Journal of Agrometeorology, Vol. 1, pp. 13-24.

El-Sherif, S. T. I., El-Areed, SH. R. M., Hagras, A. A., Mohamed, M. M. and Abd El-Hameed, A. S. (2016), "Impact of planting methods on grain yield and yield components of different bread wheat genotypes", Egyptian Journal of Plant Breeding, Vol. 20 No. 5, pp. 805-819.

FAOSTAT (2016), FAOSTAT Statistical Database, Food and Agriculture Organization of the United Nations (FAO), http://www.fao.org/giews/english/cp fs/index.htm\#2014.

Gomez, K. A. and Gomez, A. A. (1984), Statistical procedures for agricultural research, John Wiley \& Sons, New York, USA.

Hefny, Y. A. M., Dawood R. A., ElNagar, G. R. and Galal Anaam, H. (2015), "Response of two varieties productivity to planting methods and weed control under Sohag governorate conditions", Assiut Journal of Agricultural Sciences, Vol. 46 No. 3, pp. 16-28.

Ismail, A. E. A., Fakkar, A. A. O. and Hamam, K. A. (2008), "Effect of sowing methods and weed control treatments on yield and yield components of wheat", Mansoura Journal of Agricultural Science, Vol. 33 No. 3, pp. 1799-1809.

Jackson, M. L. (1973), Soil chemical 
analysis. Verlag: Prentice Hall, Inc., Englewood Cliffs, NJ. 1958, 498 S. DM 39.40.

Mahmud, M. Sh., Morsy, A. S. M. and Fakkar, A. A. O. (2016), "Evaluation of some bread wheat cultivars under different seeding rates and weed control treatments", Mansoura Journal of Plant Production, Vol. 7 No. 12, pp. 1339 -1348 .

Moagedi, A. A., Boyce, A. N. and Barakbeh, S. S. (2010), "The performance of durum and bread wheat cultivars associated with yield and yield components under different water deficit conditions", Australian of Basic and Applied Sciences, Vol. 4 No. 1, pp. 106-113.

Mohammed, B. A., Elzubeir, A. O., Hamad, M. E. and Elhagwa, A. I. (2019), "Effect of irrigation canal sediments, humate fertilizer and irrigation interval on wheat performance in desert soils", Athens Journal of Sciences, Vol. 6 No. 2, pp. 141-154.

Moustafa, A. T. H. (2013), "Effect of planting methods on grain yield and its components of some bread wheat (Triticum aestivum L.) cultivars", El-Minia Journal of Agricultural Research and Development Vol. 33 No. 4, pp. 587-595.
Na-Allah, M. S., A .Muhammad, I. U., Mohammed, T. S., Yusif Bubuche, H. and Tanimu, M. U. (2018), "Yield of wheat (Triticum aestivum L.) as influenced by planting date and planting methods in the sudan savanna ecological zone of Nigeria", International Journal of LifeSciences Scientific Research, Vol. 4 No. 5, pp. 1993-2002.

Petersen, R. G. (1985), Design and Analysis of Experiments, Marcel Dekker, Inc., New York and Basel 1985, 436 pp.

Seleiman, M. F. and Abdel-Aal, M. S. M. (2018), "Response of growth, productivity and quality of some Egyptian wheat cultivars to different irrigation regimes", Egyptian Journal of Agronomy, Vol. 40 No. 3, pp. 313-330.

Sharsher, M. S. and El-Said, S. A. (2000), "Evaluation of some wheat cultivars and lines under low and high inputs", Mansoura Journal of Agricultural Science, Vol. 25 No. 6, pp. 3109-3127.

SAS Institute (2008), The SAS system for windows release 9.2. SAS institute, Cary, N.C., USA. 